\title{
Utility of intraoperative electromyography in microvascular decompression for hemifacial spasm: a meta-analysis
}

\author{
Raymond F. Sekula Jr., M.D., Sanjay Bhatia, M.D., Andrew M. Frederickson, B.S., \\ Peter J. Jannetta, M.D., Matthew R. Quigley, M.D., George A. Small, M.D., \\ AND Ryan BREISINGER, B.S. \\ Center for Cranial Nerve Disorders, Department of Neurosurgery, Allegheny General Hospital, \\ Allegheny Neuroscience Institute/Drexel University College of Medicine, Pittsburgh, Pennsylvania
}

\begin{abstract}
Object. In this paper, the authors' goal was to determine the utility of monitoring the abnormal muscle response (AMR) or "lateral spread" during microvascular decompression surgery for hemifacial spasm.

Methods. The authors' experience with AMR as well as the data available in the English-language literature regarding resolution or persistence of AMR and the resolution or persistence of hemifacial spasm at follow-up was pooled and subjected to a meta-analysis.

Results. The pooled OR revealed by the meta-analysis was 4.2 (95\% CI 2.7-6.7). The chance of a cure if the AMR was abolished during surgery was 4.2 times greater than if the lateral spread persisted.

Conclusions. The AMR should be monitored routinely in the operating room, and surgical decision-making in the operating room should be augmented by the AMR.(DOI: 10.3171/2009.8.FOCUS09142)
\end{abstract}

\section{KEY WoRdS • hemifacial spasm • intraoperative electromyography lateral spread $\quad$ abnormal motor response}

$\mathrm{H}$ EMIFACIAL spasm, a syndrome of unilateral facial nerve hyperactive dysfunction, is a severe and disabling condition that causes impairments in the quality of life of patients. ${ }^{4}$ Typical and atypical variations of the disorder exist. In typical HFS, the spasms begin insidiously in the orbicularis oculi muscle and spread over time to muscles of the face with variable involvement of the frontalis and platysma muscles. Conversely, in atypical HFS, spasms begin insidiously in the muscles of the lower face and spread to the orbicularis oculi muscle over time. Typical HFS occurs much more frequently than atypical HFS. ${ }^{8}$

Intraoperative EMG has been used increasingly in the surgical management of HFS. ${ }^{17}$ While several retrospective and prospective studies have strongly supported its use, ${ }^{6,21,27,31}$ several other studies have failed to show that intraoperative EMG improves outcome (that is, a cure of HFS). ${ }^{1,3,9,11,28}$ To date, there are no reported randomized controlled trials of intraoperative EMG that were designed or powered to study cure of HFS as the primary end point. Because the majority of surgeons performing MVD for HFS believe strongly about the use of

\footnotetext{
Abbreviations used in this paper: $\mathrm{AMR}=$ abnormal muscle response; $\mathrm{EMG}=$ electromyography; HFS = hemifacial spasm; $\mathrm{MVD}=$ microvascular decompression .
}

intraoperative EMG, it is unlikely that a large prospective, randomized multicenter trial will be undertaken. In addition, because of the high cure rate of HFS a large number of patients will be required to uncover a difference in outcome.

In this report, we will review the salient features of EMG in HFS and our own results with intraoperative EMG. We also performed a meta-analysis of combined data regarding the utility of intraoperative EMG for MVD in HFS.

\section{Methods}

This retrospective review of existing study materials was performed under a protocol approved by our institutional review board and in accordance with patient privacy rights protection as enforced by the Health Insurance Portability and Accountability Act.

\section{Preoperative Demographics}

Between January 1, 2007, and May 31, 2009, we prospectively collected information on all patients with HFS who presented to our center. The diagnosis of HFS was based on the clinical history and physical examination and then confirmed with EMG. A Gd-enhanced MR imaging examination of the brain confirmed the absence of 
structural lesions including tumors, AVM, Chiari malformation Type I, and other confounding diagnoses (for example, multiple sclerosis) in each patient. All patients underwent preoperative EMG confirming the AMR. No patient who met these criteria was denied operation. Eighty-six patients underwent MVD by the senior authors (R.F.S. and P.J.J.). Thirteen of these patients were excluded because they had undergone a previous MVD for HFS at another institution. Additionally, intraoperative AMR data were unavailable in 4 patients because the AMR was lost after the operation was begun but before the facial nerve had been exposed. Thus, data for both the intraoperative resolution of the AMR and the postoperative outcome were available in 69 patients and were used in this analysis. In the operating room, AMR was noted as present (even if reduced by as much as 95\%) or absent (elimination of AMR).

Following MVD, data were collected at routine postoperative office visits and by formal telephone interviews of all patients in June 2009, which were conducted by a disinterested observer (A.M.F.). Each patient was evaluated in our clinic on postoperative Day 5, and all patients were queried by telephone at the last follow-up. The median follow-up was 10.7 months (range 1-28.6 months). Patients were queried regarding status of spasms immediately postoperatively and since MVD. The cases in which patients reported that their facial spasms were eliminated or were reduced by $\geq 90 \%$ (for example, occasional "fluttering about eye") were considered cured.

\section{Intraoperative EMG}

The AMR can be elicited in every patient with HFS. The term "lateral spread" is often used interchangeably with the term abnormal motor response. The term, however, should be reserved for describing the AMR in relationship to the theory of ephaptic transmission as the term "lateral spread" neglects the theory of motor nucleus hyperexcitability. To monitor the AMR intraoperatively, we use a technique similar to that originally described by Nielsen. ${ }^{25}$ Depolarizing muscle relaxants are not used. Bipolar subdermal needle electrodes are placed $0.5-1 \mathrm{~cm}$ apart in the orbicularis oculi and mentalis muscles. The orbicularis oculi muscle is activated by setting the stimulating electrode to $10 \mathrm{~mA}$ and by finding the zygomatic branches of the facial nerve supplying the ocular muscle. This is done by gently passing the stimulating electrode over the face, with slight pressure over the superior margin of the zygomatic arch. Final positioning of the stimulating electrode is based on the location that maximizes the activation of the orbicularis oculi muscle. The needle electrodes are affixed to the skin in their optimal position with tape. The AMR is recorded from the mentalis muscle. Spontaneous activity from the mentalis muscle is not usually seen with general anesthesia.

Once the AMR is obtained, the stimulating electrode is secured and a low limit threshold is obtained. The AMR is then recorded at that current intensity at $2.79 \mathrm{~Hz}$ with a $100-\mu$ sec pulse width. The stimulation frequency is $2-4 \mathrm{~Hz}$. To avoid nerve fatigue, the AMR is evoked at 5-minute intervals until dural opening. Once the dura is opened, the AMR is recorded continuously. Occasion- ally, the AMR disappears as CSF is drained or retractors are placed, ostensibly causing a relaxation of vascular compression of the facial nerve. An attempt is made to restore the AMR by increasing the current intensity (up to $15 \mathrm{~mA})$, stimulation frequency $(5.1 \mathrm{~Hz})$, and finally by increasing pulse widths in increments of $50 \mu \mathrm{sec}$. A Cadwell Cascade (Cadwell Laboratories, Inc.) machine is used at our institution.

During decompression of suspected arteries and veins from the facial nerve, the intensity of the stimulation is increased to make sure that the AMR has resolved. A further attempt is made to "drive" or stimulate the AMR by increasing the current to $20 \mathrm{~mA}$ or frequency to $30 \mathrm{~Hz}$. If the AMR cannot be driven, we consider the AMR to have completely resolved.

In addition to AMR monitoring, all patients undergoing MVD surgery are monitored for changes in the brainstem auditory evoked potentials. Occasionally direct stimulation of cranial nerve VII is used.

\section{Operative Procedure}

Microvascular decompression is performed under general anesthesia with the patient in the contralateral decubitus position, utilizing auditory brainstem evoked potentials in a previously described manner. ${ }^{15}$ A retromastoid incision is made behind the hairline, and a small craniectomy below the asterion is performed. The edge of the sigmoid sinuses is identified, and the dura mater is opened. After appropriate brain relaxation is achieved with CSF drainage, the cerebellum is gently elevated, and the root entry zone of the facial nerve is exposed and examined for vascular contact. In concert with our neuromonitoring team, arteries are decompressed from the facial nerve using shredded Teflon pledgets while some veins are coagulated using bipolar cautery or are purposely avulsed.

\section{Statistical Analysis}

Statistical analysis was performed with MedCalc 9.001 for a personal computer. Specific tests used in each instance are documented below.

\section{Results}

Our experience over the past 2 years is presented. The AMR was monitored in every patient. Seventy-three patients without previous decompressions underwent surgery performed by 2 surgeons (R.F.S. and P.J.J.). Of these, 69 patients are free of spasm at last follow-up. Two patients required reoperation for persistent spasms despite abolishment of the AMR during the first MVD. Both patients were subsequently cured of HFS with a second MVD. Both cases have been counted as failures in the meta-analysis because the patients had abolishment of the AMR at the conclusion of the first surgery, but they returned with recurrent spasms. In 4 patients, the AMR disappeared on dural opening. These 4 patients were excluded from the meta-analysis. For the purpose of the meta-analysis, 69 patients (including the 2 reoperations with subsequent cure) reported 63 cures and 6 failures 
Utility of intraoperative electromyography in MVD for HFS

TABLE 1: Intraoperative utility of the AMR

\begin{tabular}{|c|c|c|c|c|c|}
\hline \multirow[b]{2}{*}{ Authors \& Year } & \multirow[b]{2}{*}{$\begin{array}{l}\text { No. of } \\
\text { Cases }\end{array}$} & \multicolumn{4}{|c|}{ No. of Cases } \\
\hline & & $\begin{array}{l}\text { AMR Resolved/ } \\
\text { HFS Resolved }\end{array}$ & $\begin{array}{l}\text { AMR Resolved/ } \\
\text { HFS Persisting }\end{array}$ & $\begin{array}{l}\text { AMR Persisting/ } \\
\text { HFS Resolved }\end{array}$ & $\begin{array}{c}\text { AMR Persisting/ } \\
\text { HFS Persisting }\end{array}$ \\
\hline Kiya et al., 2001 & 38 & $21 / 19$ & $21 / 2$ & $17 / 11$ & $17 / 6$ \\
\hline Mooij et al., 2001 & 74 & $39 / 35$ & $39 / 4$ & $5 / 4$ & $5 / 1$ \\
\hline Hatem et al., 2001 & 33 & $23 / 20$ & $23 / 2$ & $10 / 7$ & $10 / 3$ \\
\hline Yamashita et al., 2002 & 60 & $53 / 46$ & $53 / 7$ & $6 / 5$ & $6 / 1$ \\
\hline Shin et al., 1997 & 226 & $70 / 65$ & $70 / 5$ & $62 / 44$ & $62 / 18$ \\
\hline Kong et al., 2007 & 263 & $230 / 208$ & $230 / 22$ & $33 / 22$ & $33 / 11$ \\
\hline Moller \& Jannetta, 1985 & 67 & $44 / 42$ & $44 / 2$ & $23 / 14$ & $23 / 9$ \\
\hline Isu et al., 1996 & 40 & $38 / 36$ & $38 / 2$ & $2 / 0$ & $2 / 2$ \\
\hline Joo et al., 2008 & 72 & $40 / 36$ & $40 / 4$ & $32 / 26$ & $32 / 6$ \\
\hline Huang et al., 2009 & 36 & $34 / 34$ & $34 / 0$ & $2 / 1$ & $2 / 1$ \\
\hline present study & 69 & $53 / 48$ & $53 / 5$ & $16 / 15$ & $16 / 1$ \\
\hline
\end{tabular}

with first-time MVD. The AMR (in the operating room) resolved in 53 patients. Of these, 48 patients reported no spasm on follow-up and 5 patients reported persistent spasms. Of the 69 patients included in the meta-analysis, the AMR persisted in 16 patients. In these 16 patients, the AMR was reduced by 40-95\% (except in 1 patient in whom the AMR did not change from baseline), and in 5 patients, the AMR was reduced by $>90 \%$ at the conclusion of the MVD. Of these, 15 patients reported no spasms at follow-up and 1 patient reported persistent spasms. The patient with no change in AMR from baseline, however, continues to suffer with HFS.

Since most individual studies tend to comprise a small number of patients, we have pooled the data from available studies (Table 1) and performed a meta-analysis (Fig. 1). All studies in the English-language literature (searched with Ovid Medline) with $>30$ patients, containing data on the presence or absence of the AMR at the conclusion

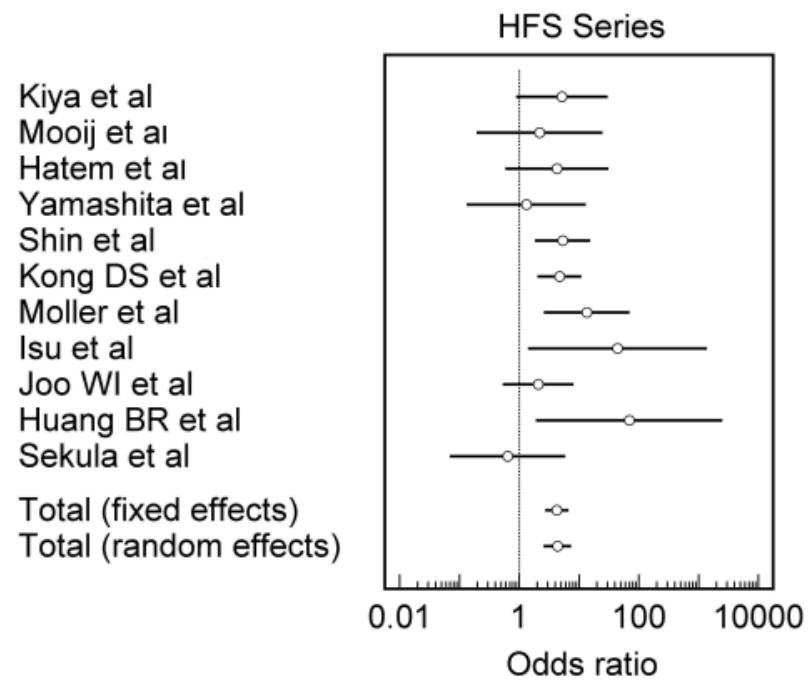

FIG. 1. Forest plot showing a meta-analysis of the various published series. Bars represent the $95 \% \mathrm{Cl}$ of the OR. The pooled estimate uses the fixed effect model as the test for heterogeneity was $p>0.05$. of MVD and presence or absence of spasms at follow-

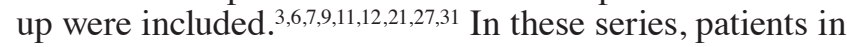
whom the AMR disappeared during dural opening or manipulation of the cerebellum were discarded from analysis. Mooij et al. ${ }^{24}$ presented their data in an intuitive fashion. For the purposes of this analysis, we included data from their confirming and inconclusive groups. Our own results have also been added to the meta-analysis (Fig. 1). Using a $2 \times 2$ contingency table and the Fisher exact test (Table 2), the pooled data revealed that a better chance of a cure with HFS is realized when the AMR is abolished during MVD ( $\mathrm{p}<0.0001)$. In decompressive surgeries in which the AMR did not resolve, the failure rate was 39\%, whereas with abolishment of the AMR the failure rate was $9.5 \%$. Resolution of the AMR reduces the failure rate almost by a factor of 4 . The collected data show a sensitivity of 0.8 , specificity of 0.53 , and accuracy of 0.8 . The pooled OR is 4.2 (95\% CI 2.7-6.7). The chance of being cured if the AMR is abolished during surgery is 4.2 times greater than when the AMR persists.

\section{Discussion}

Intraoperative EMG is simple to perform, and the AMR can be elicited in every patient with HFS. Metaanalysis of existing studies involving MVD for HFS with intraoperative EMG suggests that the rate of cure is improved when the AMR is abolished.

The diagnosis of HFS is made clinically, but EMG may help in distinguishing the disorder from other abnormal facial movements such as blepharospasm, tics, partial motor seizures, or neuromyotonia. ${ }^{14,29}$ The electrophysiological hallmarks of HFS consist of spontaneous, high-frequency (as many as 150 impulses per second), synchronized firing on EMG, and an AMR elicited with the trigeminofacial or "blink" reflex. The AMR is the recording of a response in the orbicularis oris to electrical stimuli applied over the supraorbital nerve (and sometimes a stimulus to a single facial motor branch) when the trigeminofacial reflex should be limited to the orbicularis oculi. Because of an inability to elicit the blink reflex in 
TABLE 2: Summary of published outcomes (and our experience) as a $2 \times 2$ contingency table*

\begin{tabular}{lccc}
\hline \multicolumn{1}{c}{ Variable } & HFS Resolved & HFS Persisted & Total \\
\hline AMR resolved & 589 & 56 & 645 \\
AMR persisted & 151 & 59 & 210 \\
total & 740 & 115 & 855 \\
\hline
\end{tabular}

* The Fisher exact test shows $p<0.0001$.

unconscious patients who have received doses of anesthetics compatible with those used for surgery, ${ }^{13,22-24}$ we rely on the trigeminofacial or "blink" reflex to diagnose HFS in the clinic, but we use the aforementioned technique of Nielsen ${ }^{25}$ in the anesthetized patient. Recently, however, Deletis et al. ${ }^{2}$ have proposed an anesthetic regimen that preserves the trigeminofacial or "blink" reflex. Future studies are necessary to confirm the clinical usefulness of their approach.

The trigeminofacial or "blink" reflex is based on the trigeminofacial reflex circuit. ${ }^{10}$ The afferent arc of the reflex is the trigeminal nerve, and the efferent output is produced by a pontine oligosynaptic ( 1 or 2 interneurons) pathway that give a unilateral short latency response (R1), and a spinal polysynaptic pathway (multiple interneurons) that gives bilateral long latency responses (R2 and R2C). The eventual output of both these circuits is through the efferent fibers of the facial nerve so a unilateral block in the facial nerve will cause absent $\mathrm{R} 1$ and $\mathrm{R} 2$ responses on the ipsilateral side but a normal $\mathrm{R} 2 \mathrm{C}$ response on the contralateral side. In patients with HFS, this reflex may be aberrant. As seen in this patient from our series, a larger $\mathrm{R} 1$ and R2 response (Fig. 2) is usually obtained on stimulating the affected side rather than the contralateral side (R2C), or after stimulating the unaffected side the ratio $\mathrm{R} 2 \mathrm{C} / \mathrm{R} 2$ may be increased. In HFS, the blink response may also spread to facial muscles other than the orbicularis oculi. This abnormal muscle response or "lateral spread" is an electromyographic hallmark of HFS.

In HFS, the AMR can always be recorded. However, the pathophysiological mechanism and pathological site causing the AMR are uncertain. In one proposed mechanism, the electrical stimulus activates the supraorbital nerve afferents that reach the facial motor neurons of the orbicularis oculi muscles as in the normal blink reflex. The facial nerve axons to the orbicularis oculi, however, cause a lateral spread of excitation to the axons directed to the orbicularis oris at the site of the presumed nerve demyelination (ostensibly at the area of vascular compression). ${ }^{5,26}$ A second mechanism proposes that the supraorbital nerve stimulus activates not only the facial motor neurons directed to the orbicularis oculi but also those to the orbicularis oris, due to increased facial motor neuronal excitability, facial nucleus reorganization, or even interneuronal hyperexcitability (for example, kindling). ${ }^{16}$ A third possibility is that the electrical stimulus activates not only the supraorbital nerve but also the terminal axons of the facial nerve running in the superficial facial muscles under the electrode. These would carry the volley until the site of presumed demyelination (ostensibly at

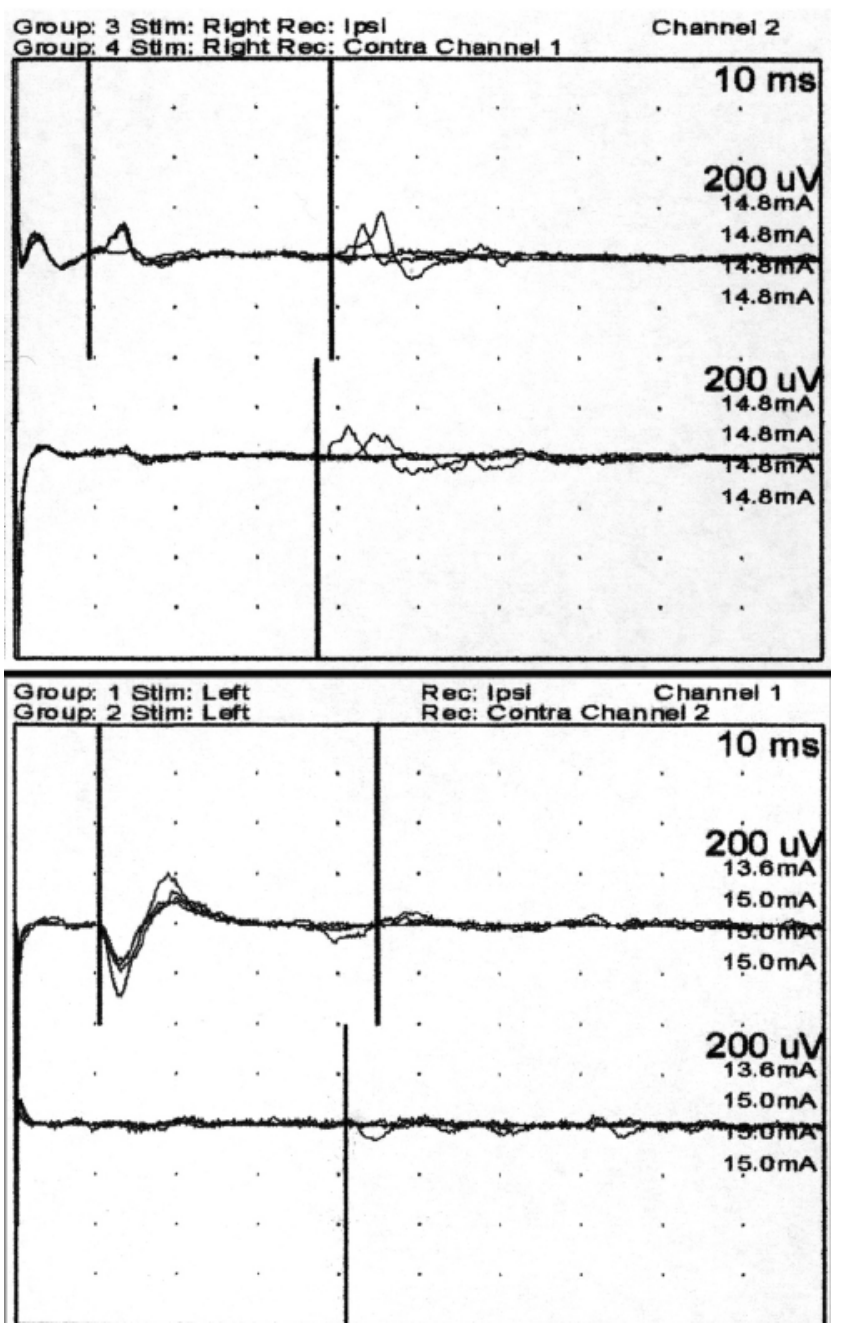

Fig. 2. Tracings. Upper: Trigeminofacial or blink reflex showing a large R2 response with stimulation of the affected side. Lower: Blink reflex showing a large R1 response with stimulation of the affected side.

the area of vascular compression) and induce the ephaptic response in the lower facial muscles. ${ }^{20}$

It is certainly possible that all 3 mechanisms contribute in part to the generation of abnormal reflex responses. As the AMR frequently disappears with vascular decompression of the facial nerve root entry zone (Fig. 3), ephaptic transmission is thought to be a more likely or a more common cause of the AMR. Isolated facial motor nucleus hyperactivity may account for a minority of HFS cases refractory to MVD or alternatively a continuum of severity of disease (that is, motor nucleus hyperexcitability follows ephaptic transmission at the site of vascular compression).

Since the AMR often disappears when the offending vessel is lifted off the facial nerve in MVD (Fig. 3), its use has been suggested as an intraoperative guide to success. ${ }^{12,17-19,31}$ Since HFS disappears or gradually resolves over time in many patients in whom the AMR persists intraoperatively, however, many authors have questioned the usefulness of intraoperative EMG. ${ }^{3,9,11,28}$ In a large study evaluating 300 patients, Kong et al. ${ }^{12}$ found a sta- 


\section{Utility of intraoperative electromyography in MVD for HFS}

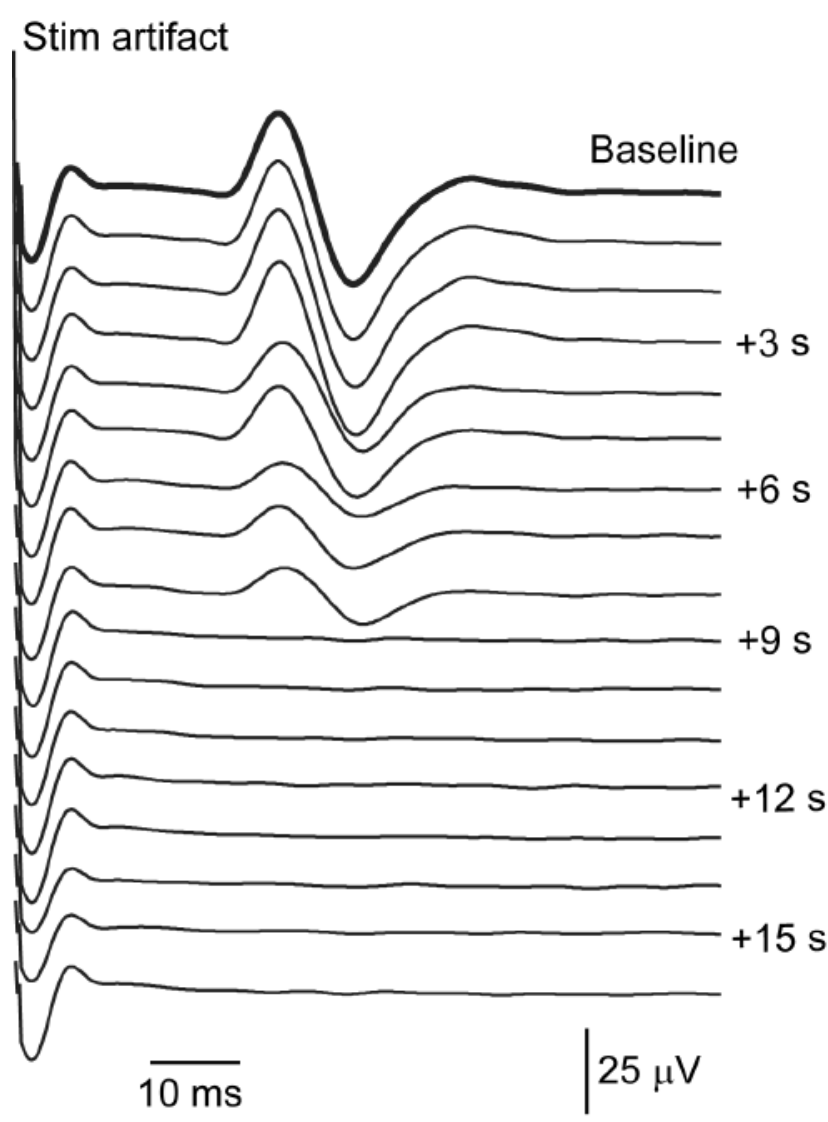

FIG. 3. Tracing showing the disappearance of the AMR with successful MVD of the facial nerve (y axis represents time in seconds following MVD of facial nerve). The compound action potential is being recorded at the mentalis muscle while the facial nerve branch to the orbicularis oculi is stimulated. Stim = stimulation.

tistical difference at the 1-year follow-up in the outcomes between 2 groups based on whether the AMR resolved or persisted. Their report is the only one that shows a statistically different outcome in cases in which the AMR did not resolve. Because of the high cure rate of this disease and the small number of patients in each series, we have pooled the data from published series and added our experience to it. Analysis of the pooled data reveals a significant difference in outcomes between the 2 groups (AMR resolved vs AMR persisted). Based on these results, an argument can be made that the AMR should be monitored routinely in the operating room, and surgical decision making in the operating room should be informed by the absence or presence of the AMR.

Limitations of our own series' results include the short duration of follow-up and a lack of postoperative EMG (that is, those patients with persistent AMR at the conclusion of surgery and continued HFS that later resolves). In the future, it may be useful to obtain EMG studies in all patients with persistent AMR at the conclusion of surgery after HFS ultimately resolves. Our rigid definition for presence (even when the AMR is reduced by $95 \%$ ) or absence of AMR may be the reason that 15 of 16 cases of patients with persistent AMR (with AMR reduction ranging between 40 and $95 \%$ ) were ultimately cured of HFS. The utility of "significant" reduction in AMR during MVD requires further study.

\section{Conclusions}

Intraoperative EMG is simple to perform, and the AMR can be elicited in every patient with HFS. Metaanalysis of existing studies involving MVD for HFS with intraoperative EMG suggests that the rate of cure is improved when the AMR is abolished.

\section{Disclaimer}

The authors report no conflict of interest concerning the materials or methods used in this study or the findings specified in this paper.

\section{References}

1. Dannenbaum M, Lega BC, Suki D, Harper RL, Yoshor D: Microvascular decompression for hemifacial spasm: long-term results from 114 operations performed without neurophysiological monitoring. J Neurosurg 109:410-415, 2008

2. Deletis V, Urriza J, Ulkatan S, Fernandez-Conejero I, Lesser J, Misita D: The feasibility of recording blink reflexes under general anesthesia. Muscle Nerve 39:642-646, 2009

3. Hatem J, Sindou M, Vial C: Intraoperative monitoring of facial EMG responses during microvascular decompression for hemifacial spasm. Prognostic value for long-term outcome: a study in a 33-patient series. Br J Neurosurg 15:496-499, 2001

4. Heuser K, Kerty E, Eide PK, Cvancarova M, Dietrichs E: Microvascular decompression for hemifacial spasm: postoperative neurologic follow-up and evaluation of life quality. Eur J Neurol 14:335-340, 2007

5. Hopf HC, Lowitzsch K: Hemifacial spasm: location of the lesion by electrophysiological means. Muscle Nerve 5 (Suppl): S84-S88, 1982

6. Huang BR, Chang CN, Hsu JC: Intraoperative electrophysiological monitoring in microvascular decompression for hemifacial spasm. J Clin Neurosci 16:209-213, 2009

7. Isu T, Kamada K, Mabuchi S, Kitaoka A, Ito T, Koiwa M, et al: Intra-operative monitoring by facial electromyographic responses during microvascular decompressive surgery for hemifacial spasm. Acta Neurochir (Wien) 138:19-23, 1996

8. Jannetta PJ: Typical or atypical hemifacial spasm. J Neurosurg 89:346-347, 1998

9. Joo WI, Lee KJ, Park HK, Chough CK, Rha HK: Prognostic value of intra-operative lateral spread response monitoring during microvascular decompression in patients with hemifacial spasm. J Clin Neurosci 15:1335-1339, 2008

10. Kimura J, Powers JM, Van Allen MW: Reflex response of orbicularis oculi muscle to supraorbital nerve stimulation. Study in normal subjects and in peripheral facial paresis. Arch Neurol 21:193-199, 1969

11. Kiya N, Bannur U, Yamauchi A, Yoshida K, Kato Y, Kanno T: Monitoring of facial evoked EMG for hemifacial spasm: a critical analysis of its prognostic value. Acta Neurochir (Wien) 143:365-368, 2001

12. Kong DS, Park K, Shin BG, Lee JA, Eum DO: Prognostic value of the lateral spread response for intraoperative electromyography monitoring of the facial musculature during microvascular decompression for hemifacial spasm. J Neurosurg 106:384-387, 2007

13. Marelli RA, Hillel AD: Effects of general anesthesia on the human blink reflex. Head Neck 11:137-149, 1989

14. Marti-Fabregas J, Montero J, Lopez-Villegas D, Quer M: Post- 
irradiation neuromyotonia in bilateral facial and trigeminal nerve distribution. Neurology 48:1107-1109, 1997

15. McLaughlin MR, Jannetta PJ, Clyde BL, Subach BR, Comey $\mathrm{CH}$, Resnick DK: Microvascular decompression of cranial nerves: lessons learned after 4400 operations. J Neurosurg 90:1-8, 1999

16. Moller AR: Hemifacial spasm: ephaptic transmission or hyperexcitability of the facial motor nucleus? Exp Neurol 98:110-119, 1987

17. Moller AR, Jannetta PJ: Hemifacial spasm: results of electrophysiologic recording during microvascular decompression operations. Neurology 35:969-974, 1985

18. Moller AR, Jannetta PJ: On the origin of synkinesis in hemifacial spasm: results of intracranial recordings. J Neurosurg 61:569-576, 1984

19. Moller AR, Jannetta PJ: Physiological abnormalities in hemifacial spasm studied during microvascular decompression operations. Exp Neurol 93:584-600, 1986

20. Montero J, Junyent J, Calopa M, Povedano M, Valls-Sole J: Electrophysiological study of ephaptic axono-axonal responses in hemifacial spasm. Muscle Nerve 35:184-188, 2007

21. Mooij JJ, Mustafa MK, van Weerden TW: Hemifacial spasm: intraoperative electromyographic monitoring as a guide for microvascular decompression. Neurosurgery 49:1365-1371, 2001

22. Mourisse J, Gerrits W, Lerou J, van Egmond J, Zwarts MJ, Booij L: Electromyographic assessment of blink and corneal reflexes during midazolam administration: useful methods for assessing depth of anesthesia? Acta Anaesthesiol Scand 47:593-600, 2003

23. Mourisse J, Lerou J, Struys M, Zwarts M, Booij L: Multilevel approach to anaesthetic effects produced by sevoflurane or propofol in humans: 2. BIS and tetanic stimulus-induced withdrawal reflex. Br J Anaesth 98:746-755, 2007

24. Mourisse J, Lerou J, Zwarts M, Booij L: Electromyographic assessment of blink reflexes correlates with a clinical scale of depth of sedation/anaesthesia and BIS during propofol administration. Acta Anaesthesiol Scand 48:1174-1179, 2004

25. Nielsen VK: Electrophysiology of the facial nerve in hemifacial spasm: ectopic/ephaptic excitation. Muscle Nerve 8:545555,1985

26. Nielsen VK: Pathophysiology of hemifacial spasm: II. Lateral spread of the supraorbital nerve reflex. Neurology 34:427431,1984

27. Shin JC, Chung UH, Kim YC, Park CI: Prospective study of microvascular decompression in hemifacial spasm. Neurosurgery 40:730-735, 1997

28. Sindou MP: Microvascular decompression for primary hemifacial spasm. Importance of intraoperative neurophysiological monitoring. Acta Neurochir (Wien) 147:1019-1026, 2005

29. Valls-Sole J: Facial palsy, postparalytic facial syndrome, and hemifacial spasm. Mov Disord 17 (Suppl 2):S49-S52, 2002

30. Yamashita S, Kawaguchi T, Fukuda M, Suzuki K, Watanabe M, Tanaka R, et al: Lateral spread response elicited by double stimulation in patients with hemifacial spasm. Muscle Nerve 25:845-849, 2002

31. Yamashita S, Kawaguchi T, Fukuda M, Watanabe M, Tanaka R, Kameyama S: Abnormal muscle response monitoring during microvascular decompression for hemifacial spasm. Acta Neurochir (Wien) 147:933-938, 2005

Manuscript submitted June 15, 2009.

Accepted August 19, 2009.

Address correspondence to: Raymond F. Sekula Jr., M.D., Center for Cranial Nerve Disorders, Department of Neurosurgery, Allegheny General Hospital, 420 East North Avenue, Suite 302, Pittsburgh, Pennsylvania 15212. email: rsekula@wpahs.org. 